\title{
Influenza A Infection Triggers Thrombotic Thrombocytopenic Purpura by Producing the Anti-ADAMTS13 IgG Inhibitor
}

\author{
Nobuharu Kosugi ${ }^{1}$, Yuya Tsurutani ${ }^{1}$, Ayami Isonishi ${ }^{2}$, Yuji Hori ${ }^{2}$, \\ Masanori Matsumoto $^{2}$ and Yoshihiro Fujimura ${ }^{2}$
}

\begin{abstract}
A 68-year-old Japanese woman infected with influenza A developed thrombotic thrombocytopenic purpura (TTP) 2 days after having a fever. Routine laboratory tests on admission suggested a diagnosis of disseminated intravascular coagulation. However, ADAMTS13 assays showed an extremely low level of plasma ADAMTS13 activity with a high titer of anti-ADAMTS13 inhibitor ( $\mathrm{IgG})$. Despite high-dose methylprednisolone therapy with daily plasma exchange for 3 consecutive days, the patient died of pulmonary congestion complicated by cardiac failure. Our experience here provides the first evidence that influenza A infection is sufficient to trigger TTP by producing the anti-ADAMTS13 IgG inhibitor.
\end{abstract}

Key words: influenza, TTP

(Inter Med 49: 689-693, 2010)

(DOI: 10.2169/internalmedicine.49.2957)

\section{Introduction}

Thrombotic thrombocytopenic purpura (TTP) is a rare but life-threatening disease, characterized by generalized microvascular occlusion by platelet thrombi $(1,2)$. TTP was classically identified by a clinical 'pentad' consisting of thrombocytopenia, microangiopathic hemolytic anemia, renal failure, fever, and fluctuating neurological signs (3). More recently, however, TTP, has been associated with a deficiency of plasma ADAMTS13 (a disintegrin-like metalloproteinase with thrombospondin type 1 motifs 13) activity, which is caused by genetic mutations in or acquired autoantibodies to this enzyme.

ADAMTS13 specifically cleaves von Willebrand factor multimers (VWFMs) at the site of the Tyr1605-Met1606 bond of the VWF-A2 domain (4). In the absence of ADAMTS13 activity, therefore, unusually large VWFMs (UL-VWFMs) are produced in the vascular endothelial cells, left uncleaved, and released into the circulation. The accumulated UL-VWFMs in the circulation induce generalized formation of platelet thrombi in the microvasculatures under certain rheological conditions, resulting in TTP. Acquired TTP may develop under various clinical conditions, such as drug use (5), pregnancy, malignancies, and collagen diseases (6), as typically seen in systemic lupus erythematosus. It has also been reported that acquired deficiency of ADAMTS13 activity is closely associated with human immunodeficiency virus (HIV) infection (7) and influenza vaccination with or without adjuvants (8-10), but has not been reported to be associated with influenza infection alone. In this paper, we describe the case of an acquired TTP patient with severe ADAMTS13 activity deficiency due to neutralizing IgG-autoantibodies, manifested as a serious complication from influenza A infection.

\section{Case Report}

In early March, 2007, a 68-year-old woman weighing 50 $\mathrm{kg}$ visited a local physician with complaints of fever and general malaise. On the following day a diagnosis of influenza A was made from throat swab specimens using the

${ }^{1}$ Department of Internal Medicine, Numazu City Hospital, Numazu and ${ }^{2}$ Department of Blood Transfusion Medicine, Nara Medical University, Kashihara

Received for publication October 1, 2009; Accepted for publication December 15, 2009

Correspondence to Dr. Yoshihiro Fujimura, malon@ naramed-u.ac.jp 
Table 1. Laboratory Findings on Admission

\begin{tabular}{|c|c|c|}
\hline \multicolumn{2}{|l|}{ Peripheral blood } & (Control) \\
\hline Platelets $\left(\times 10^{6} / \mathrm{L}\right)$ & 6 & $(140-360)$ \\
\hline White blood cell $\left(\times 10^{3} / \mathrm{L}\right)$ & 7200 & $(3500-9500)$ \\
\hline Red blood cell $\left(\times 10^{6} / \mathrm{L}\right)$ & 222 & (F360-500) \\
\hline Hemoglobin $(\mathrm{g} / \mathrm{dL})$ & 6.6 & $(\mathrm{~F} 11.5-15.5)$ \\
\hline Reticulocyte (\%o) & 35 & $(0.5-1.5)$ \\
\hline Schistocytes on blood film & +++ & $(-)$ \\
\hline \multicolumn{3}{|l|}{ Blood chemistry } \\
\hline Total protein $(\mathrm{g} / \mathrm{dL})$ & 5.1 & $(6.5-8.2)$ \\
\hline Total bilirubin (mg/dL) & 2.9 & $(0.2-1.1)$ \\
\hline Direct bilirubin (mg/dL) & 0.8 & $(0-0.3)$ \\
\hline Aspartate aminotransferase (IU/L) & 694 & $(8-40)$ \\
\hline Alanine aminotransferase (IU/L) & 399 & $(3-35)$ \\
\hline Lactate dehydrogenase (IU/L) & 3060 & $(119-229)$ \\
\hline Blood urea nitrogen $(\mathrm{mg} / \mathrm{dL})$ & 88 & $(7-22)$ \\
\hline Creatinine $(\mathrm{mg} / \mathrm{dL})$ & 2.95 & $(0.4-1.2)$ \\
\hline \multicolumn{3}{|l|}{ Cytokines } \\
\hline TNF- $\alpha(\mathrm{pg} / \mathrm{mL})$ & 3.54 & $(<1.79)$ \\
\hline $\mathrm{IL}-6(\mathrm{pg} / \mathrm{mL})$ & 12.8 & $(<2.41)$ \\
\hline $\mathrm{IL}-8(\mathrm{pg} / \mathrm{mL})$ & 28.7 & $(<8.0)$ \\
\hline C-reactive protein (mg/dL) & 9.1 & $(<0.5)$ \\
\hline \multicolumn{3}{|l|}{ Hemostatic test } \\
\hline PT (sec) & 16.9 & $(11-12)$ \\
\hline A-PTT (sec) & 33 & $(21.0-38.0)$ \\
\hline Fibrinogen (mg/dL) & 552 & $(150-400)$ \\
\hline Antithrombin (\%) & 72 & $(80-130)$ \\
\hline Plasminogen (\%) & 79 & $(66-130)$ \\
\hline a2-Plasmin inhibitor (\%) & 42.99 & $(90-140)$ \\
\hline FDP-P (ug/mL) & 71 & $(<5)$ \\
\hline D-dimer (ug/mL) & 23.14 & $(<1.0)$ \\
\hline TAT (ng/mL) & 11.8 & $(<3)$ \\
\hline $\mathrm{PIC}(\mathrm{ug} / \mathrm{mL})$ & 2.4 & $(<1.0)$ \\
\hline VWF antigen (\%) & 184 & $(50-150)$ \\
\hline \multicolumn{3}{|l|}{ ADAMTS13 } \\
\hline Activity (\%) & $<0.5$ & $(50-150)$ \\
\hline Antigen (\%) & 0.1 & $(50-150)$ \\
\hline Inhibitor (Bethesda U/mL) & 6.0 & $(<0.5)$ \\
\hline
\end{tabular}

immuno-chromatography method. She received oral administration of oseltamivir (150 mg/day). Previously, she had had surgeries for a gastric cancer (47 y.o.) and uterine myoma (48 y.o.) without transfusion. She had not had either bleeding diathesis or thrombotic tendencies before. Two days later, her general condition worsened, and she was admitted to a nearby hospital, where TTP was suspected from clinical signs and the results of routine laboratory tests. Therefore, she was referred to our hospital for plasma exchange therapy the following day. Laboratory results before the plasma exchange are shown in Table 1. Tests for a series of autoantibodies related to collagen diseases and for antibodies to HIV and hepatitis $\mathrm{C}$ were all negative. However, she tested positive for antibodies against both hepatitis B surface and core antigens, but negative for surface antigen. Further, the plasma levels of tumor necrosis factor (TNF)- $\alpha$, interleukin (IL)-6, IL-8, and C-reactive protein (CRP) were high. The patient had the typical clinical signs of TTP ('pentad'), thus a clinical diagnosis of TTP was made without difficulty; however, retrospectively, it was interesting to note the following laboratory results, which slightly resem- bled what would be expected for disseminated intravascular coagulation (DIC): 1) prolonged prothombin time (PT), despite normal activated partial prothrombin time (A-PTT); 2) significantly reduced plasma level of antithrombin; 3) increased levels of plasma fibrinogen and two of its degradation products, FDP-P and D-dimer; 4) elevated levels of the DIC markers, thrombin-antithrombin complex (TAT) and plasmin- $\alpha 2$ plasmin inihibitor complex (PIC). Thus, by the diagnostic criteria for DIC from the International Society of Thrombosis and Haemostasis (11), the patient had a DIC score of 4 (non-overt DIC), and by that from the Japanese Ministry of Health and Welfare (12), the patient had a DIC score of 9 (overt DIC). However, ADAMTS13 assays in this patient gave evidence confirming a diagnosis of TTP, showing an extremely low level of plasma ADAMTS13 activity (less than $0.5 \%$ of the normal control) with a high titer of anti-ADAMTS13 inhibitor (6.0 Bethesda units/mL), using chromogenic act-ELISA (13). The plasma level of ADAMTS13 antigen was $0.1 \%$ by ag-ELISA (14).

After admission, the patient received high-dose methylprednisolone (one gram per day) therapy with concomitant 


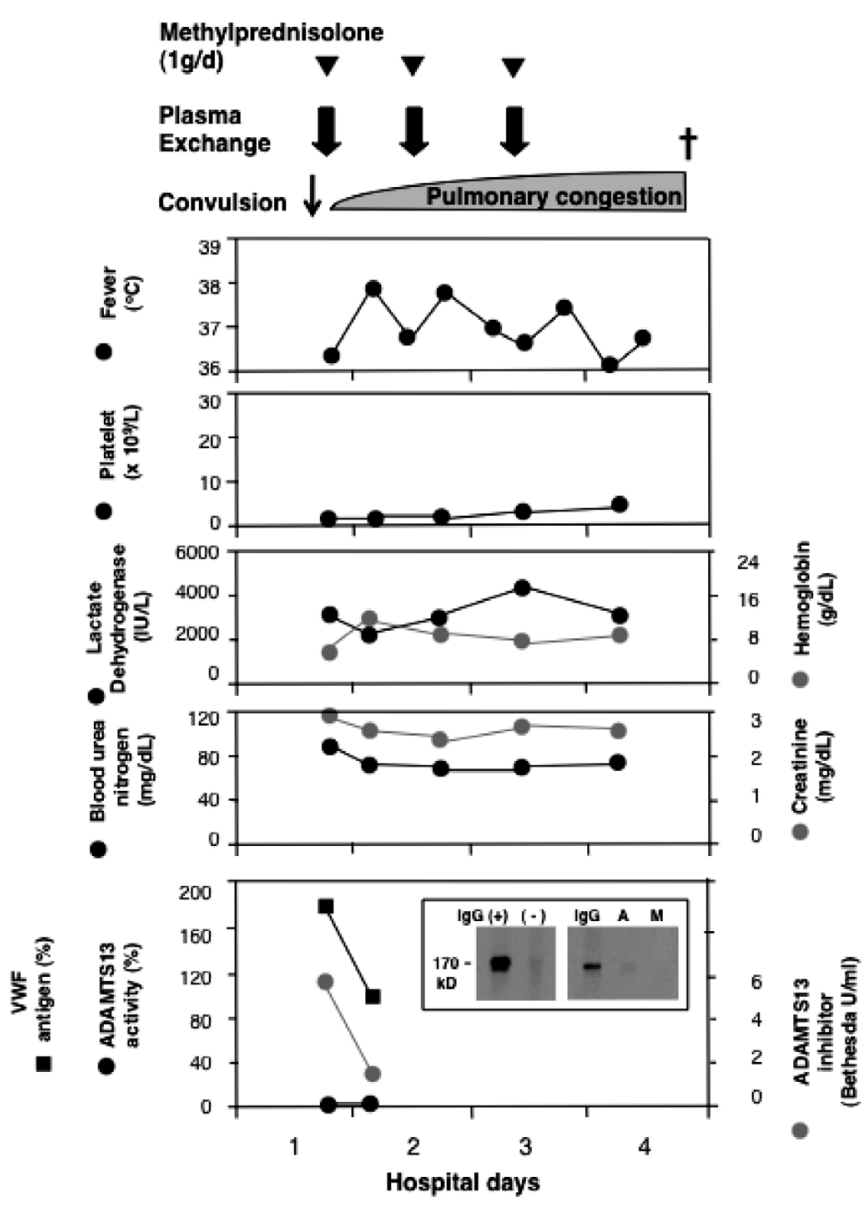

Figure 1. Clinical course of influenza A-associated thrombotic thrombocytopenic purpura (TTP). A 68-year-old Japanese woman infected with influenza A developed TTP 2 days after having a fever. Plasma ADAMTS13 activity was less than $0.5 \%$ of normal control, and a high titer of antiADAMTS13 inhibitor (6.0 Bethesda units/mL) was measured. Western blot studies using purified plasma-derived ADAMTS13 revealed that the immunoglobulin type of this inhibitor was IgG (inset). As shown in the left panel of inset (bottom figure), heated plasma from the patient with acquired idiopathic TTP and IgG inhibitors against ADAMTS13 (+) displayed a 170 kD-band as a positive control, while that from a normal individual without ADAMTS13 inhibitor (-) showed no band (a negative control). In the right panel of inset, the heated plasma of this patient displayed the band detected by anti-human IgG polyclonal antibody, but not by anti-human IgA, or IgM polyclonal antibody.

daily plasma exchange at a single dose volume of 3,240$3,600 \mathrm{~mL}$ for 3 consecutive days. Red blood cell concentrates were also transfused with a total volume of $840 \mathrm{~mL}$, but no infusion of platelet concentrates was performed throughout the clinical course. On the day after admission to our hospital, despite this intensive therapy, the patient developed generalized convulsion, which were controlled by parenteral administration of anti-convulsant (aleviatin). Never- theless, pulmonary congestion developed, which was presumably induced by hypoproteinemia, and necessitated the use of anti-diuretics. Two days later, the patient's electrocardiogram suddenly showed signs of cardiac ischemia/infarction (elevation of the ST-wave), and 3 hours later she died. Autopsy was not performed.

Western blot studies of the patient's plasma, which had been stored at $-80^{\circ} \mathrm{C}$, and the purified plasma-derived ADAMTS13, clearly identified that the immunoglobulin type of the inhibitor was $\operatorname{IgG}$ (6) (Fig. 1, inset).

\section{Discussion}

In 1981, Wasserstein et al (15), described a case of a 50year-old man with recurrent TTP which manifested solely as aphasia after influenza infection; however, clinical diagnosis of TTP was not made during the acute phase in this patient. During the chronic phase the diagnosis was made by renal biopsy demonstrating the features of chronic renal disease: glomerulus showing thickened capillary wall with numerous 'double contours' as well as several hyaline capillary thrombi, accompanied by microangipathic hemolytic anemia and thrombocytopenia. This paper is the first to describe a close relationship between influenza and TTP, but without addressing pathogenesis.

A relationship between the influenza 'swine-flu' vaccine and an increased risk of Guillain-Barré syndrome was reported in 1991 (16), but the relationship with thrombocytopenia was not shown. In recent years, two groups of investigators $(8,9)$ have reported that the inactivated human influenza vaccine may induce TTP bouts by boosting the production of anti-ADAMTS13 autoantibodies. To date, however, autoantibody-induced TTP has never been reported in association with the influenza infection itself. In this regard, the present report provides the first evidence that influenza A infection alone may trigger TTP by the production of anti-ADAMTS13 IgG. The question remains: why and how has this effect been hidden to date? Is it a new or extremely rare phenomenon among patients infected with influenza A?

In this regard, we are now particularly interested in influenza-associated encephalopathy (IAE), which has been reported to occur at a frequency of $0.02 \%$ among Japanese patients infected with influenza A and/or B. More interestingly, IAE has rarely been reported in the United States or in European countries (17), a fact which we cannot explain. We do, however, note several important phenomena (17). First, IAE develops either on the day that influenza signs appear or on the following day, and is highly heterogeneous in terms of neurological findings. Second, influenza virus can seldom be identified in the cerebrospinal fluid or brain tissues in the affected individuals. Third, influenza infection is often complicated by pneumonia and less commonly by IAE, both of which are occasionally associated with multiorgan failure (MOF). The MOF in these settings has been explained by DIC-like pathogenesis generated by the ex- 
tremely high plasma levels of various cytokines, termed a 'cytokine storm,' which injures vascular endothelial cells where platelet thrombi are likely to be formed. In fact, the platelet count is correlated with the outcome of IAE; of the patients with thrombocytopenia $(<50,000$ platelets/uL), more than $80 \%$ died. Fourth, generalized microemboli and hyalinization of the small vessels were observed in autopsies (17). Finally, some patients with IAE have responded well to high-dose steroid therapy with plasma exchange (18). This therapeutic efficacy has been attributed to the resolution of the plasma 'cytokine storm', but the precise mechanism remains undetermined. Thus, we find a high similarity between IAE and influenza A-associated TTP, although they are not identical.

The present case was diagnosed as acquired TTP by severe deficiency of ADAMTS13:AC with anti-ADAMTS13 inhibitor, but also fulfilled the diagnostic criteria for DIC by the Japanese Ministry of Health and Welfare (12). In this regard, we can address the followings: TMAs are often indistinguishable from DIC by clinical signs alone, but now it is well accepted that TMAs are pathologically featured by platelet thrombi and DIC by fibrin thrombi, each formed in microvasculatures. Thus, it is conceivable that TMA is further complicated by DIC and both clinical conditions may co-exist, but its reversal clinical course appears to be less likely.

In our database of Japanese patients (19), 69\% (195/284) of patients with acquired idiopathic TTP also had a severe deficiency of ADAMTS13 activity caused by the development of anti-ADAMTS13 autoantibodies (IgG), implying that acquired idiopathic TTP is an autoimmune disease. Consistent with this, the present case report implies that influenza A infection triggers TTP which is almost indistinguishable from acquired idiopathic TTP by producing antiADAMTS13 inhibitory IgG. To determine a possible association between influenza-associated TTP and IAE would be of great interest in future studies. Our experience shows the necessity of the analysis of ADAMTS13 activity and its inhibitors to differentiate between clinical conditions and to determine a therapeutic strategy for influenza infection.

\section{Acknowledgement}

The authors would like to thank Dr. Jan Voorberg of Sanquin for his valuable comments and suggestions in preparing this manuscript. The authors also thank Mitsubishi Chemical Medience (Tokyo, Japan) for determining plasma levels of cytokines (IL-6, IL-8, and TNF $\alpha$ ).

\section{Grant Support}

This work was supported in part by research grants from the Ministry of Education, Culture, Sports, Science and Technology of Japan and from the Ministry of Health, Labor, and Welfare of Japan (Director: Dr. Mitsuru Murata).

\section{References}

1. Moschcowitz E. Hyaline thrombosis of the terminal arterioles and capillaries: A hitherto undescribed disease. Proc N Y Pathol Soc 24: 21-24, 1924.

2. George JN. Clinical practice. Thrombotic thrombocytopenic purpura. N Engl J Med 354: 1927-1935, 2006.

3. Amorosi EL, Ultmann JE. Thrombotic thrombocytopenic purpura: Report of 16 cases and review of the literature. Medicine 45: 139159, 1966.

4. Sadler JE. Von Willebrand factor, ADAMTS13, and thrombotic thrombocytopenic purpura. Blood 112: 11-18, 2008.

5. Zakarija A, Bennett C. Drug-induced thrombotic microangiopathy. Semin Thromb Hemost 31: 681-690, 2005.

6. Matsuyama T, Kuwana M, Matsumoto M, Isonishi A, Inokuma S, Fujimura Y. Heterogeneous pathogenic processes of thrombotic microangiopathies in patients with connective tissue diseases. Thromb Haemost 102: 371-378, 2009.

7. Bell WR, Chulay JD, Feinberg JE. Manifestations resembling thrombotic microangiopathy in patients with advanced human immunodeficiency virus (HIV) disease in a cytomegalovirus prophylaxis trial (ACTG 204). Medicine (Baltimore) 76: 369-380, 1997.

8. Brodin-Sartorius A, Guebre-Egziabher F, Fouque D, et al. Recurrent idiopathic thrombotic thrombocytopenic purpura: A role of vaccination in disease relapse? Am J Kidney Disease 48: e31-e34, 2006.

9. Dias PJ, Gopal S. Refractory thrombotic thrombocytopenic purpura following influenza vaccination. Anaesthesia 64: 444-446, 2009.

10. Wang T-T, Palese P. Universal epitopes of influenza virus hemagglutinins? Nature Struc Mol Biol 16: 233-234, 2009.

11. Taylor FB Jr, Tob CH, Hoots WK, et al. Towards definition, clini- cal and laboratory criteria, and a scoring system for disseminated intravascular coagulation-On behalf of the Scientific Subcommittee on disseminated intravascular coagulation (DIC) of the International Society on Thrombosis and Hamostasis (ISTH). Thromb Haemost 86: 1327-1330, 2001.

12. Wada $H$, Wakita $Y$, Nakase $T$, et al. Increased plasma-soluble fibrin monomer levels in patients with disseminated intravascular coagulation. Am J Hematol 51: 255-260, 1996.

13. Kato $S$, Matsumoto M, Matsuyama $T$, Isonishi A, Hiura H, Fujimura Y. Novel monoclonal antibody-based immunoassay for determining plasma levels of ADAMTS13 activity. Transfusion 46: 1444-1452, 2006.

14. Yagi H, Ito S, Kato S, Hiura $H$, Matsumoto M, Fujimura M. Plasma levels of ADAMTS13 antigen, determined by an enzyme immunoassay using the neutralizing monoclonal antibody, parallel to those of the activity. Int J Hematol 85: 403-407, 2007.

15. Wasserstein A, Gary H, Goldfarb S, Goldberg M. Recurrent thrombotic thrombocytopenic purpura after viral infection. Arch Intern Med 141: 685-687, 1981.

16. Safranek TJ, Lawrence DN, Kurland LT, et al. Reassessment of the association between Guillain-Barré syndrome and receipt of swine influenza vaccine in 1976-1977: results of a two-state study. Expert Neurology Group. Am J Epidemiol 133: 940-951, 1991.

17. Morishima T, Togashi $T$, Yokota $S$, et al. Encephalitis and encephalopathy associated with an influenza epidemic in Japan. Clin Infect Dis 35: 512-517, 2002.

18. Kawashima H, Togashi T, Yamanaka G, et al. Efficacy of plasma exchange and methylpredonisolone pulse thrapy on influenzaassociated encephalopathy. J Infect 51: e53-e56, 2005.

19. Fujimura Y, Matsumoto M. Registry of 919 patients with throm- 
Inter Med 49: 689-693, 2010 DOI: 10.2169/internalmedicine.49.2957

botic microangiopathies across Japan: Database of Nara Medical University during 1998-2008. Intern Med 49: 7-15, 2010.

(C) 2010 The Japanese Society of Internal Medicine http://www.naika.or.jp/imindex.html 